\title{
Los estudios doctorales: crecimiento profesional por sobre los incentivos organizacionales. Un estudio en la Universidad Estatal a Distancia (UNED) de Costa Rica
}

\author{
Karla Yanitzia Artavia Díaz ${ }^{1}$, Alejandra Castro Granados ${ }^{2}$ \\ ${ }^{1}$ Universidad Estatal a Distancia - UNED. Programa de Investigación en Fundamentos de Educación a Distancia (PROIFED). \\ Sabanilla, Mercedes de Montes de Oca. San José, Costa Rica. ${ }^{2}$ Universidad Estatal a Distancia - UNED. Programa de \\ Aprendizaje en Línea (PAL) de la Universidad Estatal a Distancia. \\ Autor para correspondência/Author for correspondence: kartavia@uned.ac.cr
}

RESUMEN. La generación de conocimiento es un quehacer necesario e indispensable de la educación superior, que requiere de una constante actualización, así como de un compromiso por mejorar el mundo actual. Sin embargo, ello implica que exista un verdadero interés por la formación profesional y con ello la posible obtención de un grado académico de doctorado, más allá de los incentivos organizacionales que con dicha titulación se puedan obtener, ya sea que se otorguen o no. Para conocer al respecto, se realizó un estudio en la Universidad Estatal a Distancia (UNED) de Costa Rica con el objetivo de analizar el interés para la formación de alto nivel en investigación, conducente a un doctorado académico según los diferentes campos disciplinares; a 892 funcionarios que cuentan con título de maestría y de los cuales se obtuvo respuesta de 304 personas, fundamentado en un paradigma naturalista con enfoque cuantitativo, para lo cual se aplicó una encuesta de opinión para la recolección de la información. El estudio logró determinar que las recompensas o factores de motivación con mayor apreciación por parte de los participantes se relacionan, en su mayoría, con el planteamiento de proyectos con beneficio social, así como la vinculación a diferentes redes de investigación tanto nacionales como internacionales dejando relegada la opción relacionada con el posible aumento salarial.

Palabras clave: Organizacionales, Recompensas Personales, Doctorado, Crecimiento Profesional-académico-investigativo, Motivación, Intereses.

\begin{tabular}{|l|l|l|l|l|l|l|} 
RBEC & Tocantinópolis/Brasil & v. 5 & e8519 & $10.20873 /$ uft.rbec.e8519 & 2020 & ISSN: 2525-4863 \\
\hline
\end{tabular}




\title{
Doctoral studies: professional growth over organizational incentives. A study at the Universidad Estatal a Distancia (UNED) from Costa Rica
}

\begin{abstract}
The generation of knowledge is a necessary and indispensable task of higher education, which requires constant updating as well as a commitment to improve the real world. However, this implies that there is a real interest in professional training and thus the possible obtaining of an academic doctorate degree, beyond the organizational incentives that can be obtained with such qualification, whether or not they are granted. To learn about this, a study was carried out at the Universidad Estatal a Distancia (UNED) of Costa Rica with the aim of analyzing the interest for high-level training in research, leading to an academic doctorate according to the different disciplinary fields; to 892 officials with a master's degree and of whom a response was obtained from 304 persons; based on a naturalistic paradigm with a quantitative approach, for which an opinion survey was applied for the collection of information. The study found that the rewards or motivation factors with the greatest appreciation by the participants relate, for the most part, to the project approach with social benefit, as well as the linking to different national and international research networks, leaving aside the option related to the possible wage increase.
\end{abstract}

Keywords: Organizational Incentives, Personal Rewards, Doctorate Degree, Professional-academic-research growth, Motivation, Interests. 


\section{Estudos de doutorado: crescimento profissional sobre incentivos organizacionais. Um estudo da Universidad Estatal a Distancia (UNED) da Costa Rica}

RESUMO. A geração de conhecimento é uma tarefa necessária e indispensável do ensino superior, que exige atualização constante, bem como o compromisso de melhorar o mundo atual. No entanto, isso implica que existe um interesse real na formação profissional e, com isso, a possível obtenção de um diploma acadêmico de doutorado além dos incentivos organizacionais que podem ser obtidos com esse diploma, sejam concedidos ou não. Para se ter uma noção, foi realizado um estudo na Universidad Estatal a Distancia (UNED) da Costa Rica com o objetivo de analisar o interesse pela formação de alto nível em pesquisa, levando a um doutorado acadêmico de acordo com as diferentes áreas disciplinares; para 892 funcionários com mestrado, foi obtida uma resposta de 304 pessoas, baseado em um paradigma naturalista com abordagem quantitativa, para o qual foi aplicada uma pesquisa de opinião para a coleta de informações. O estudo foi capaz de determinar que as recompensas ou fatores motivadores de maior valorização pelos participantes estão principalmente relacionados à abordagem de projetos com benefício social, bem como ao vínculo com diferentes redes de pesquisa nacionais e internacionais, deixando relegada a opção relacionada ao possível aumento salarial.

Palavras-chave: Incentivos Organizacionais, Recompensas Pessoais, $\mathrm{PhD}$, Crescimento da Pesquisa profissional-acadêmica, Motivação, Interesses. 


\section{Introducción}

El tema de los incentivos $\mathrm{y}$ recompensas ha comenzado a tener un mayor impacto en la cultura académica y de investigación, esto debido al interés constante de superación personal, profesional y académica en las nuevas dinámicas de la cultura organizacional. Cada vez son más las teorías de motivación e incentivos organizacionales que se aplican en las instituciones para que los empleados mantengan el interés y desarrollen su trabajo con eficiencia y eficacia.

Tanto para los empleadores como para los empleados, la formación académica es un tema muy relevante debido a la adquisición y actualización de conocimiento en beneficio de todos los procesos productivos; por lo que las Universidades, como entes encargadas de impartir educación de calidad a la sociedad, se preocupan aún más al respecto, de manera que sus académicos e investigadores cuenten con un grado académico doctoral que les brinde mayor reconocimiento y prestigio, así como la posibilidad de participar en más y mejores redes $\mathrm{y}$ proyectos nacionales $\mathrm{e}$ internacionales.

Si bien la obtención de dicha titulación es una meta profesional para muchas personas debido a los posibles beneficios que se obtienen como por ejemplo, la generación y participación en redes, trabajo colaborativo interdisciplinario, liderazgo en áreas de investigación e innovación, adquisición de recursos para concreción de proyectos, participación en congresos como experto, y la posible obtención de un ascenso profesional entre otros; sin embargo, esto no es una garantía explícita en muchas instituciones y aunado a una serie de sacrificios por realizar de parte del educando en diferentes áreas personales y profesionales, todo ello puede llegar a convertirse en un factor de riesgo que ocasione la pérdida del interés en optar por dicha titulación.

En así como las instituciones de educación superior se encuentran ante un gran reto, la atracción de personal interesado en cursar doctorados, para lo que se necesita de propuestas estratégicas que deriven en incentivos importantes que motiven seriamente a su incursión, de manera que quienes comiencen este recorrido se mantengan firmes y logren culminar con éxito sin perder el interés.

Con respecto a lo anterior, se plantea una investigación con el objetivo de identificar el interés por la formación de alto nivel en investigación conducente al doctorado académico en diferentes campos disciplinares, de funcionarios de la 
Universidad Estatal a Distancia (UNED) de Costa Rica; partiendo de la serie de incentivos económicos y recompensas personales que existen en la Universidad.

Para ello se plantearon algunas interrogantes que orientaron la obtención de los datos que permitieran dar respuesta al objetivo planteado, entre las que se encuentran: ¿cuáles condiciones laborales necesitaría para poder vincularse, eventualmente, a un programa de formación?, ¿en cuál modalidad estaría interesada(o) en llevar el programa de formación?, ¿cuáles serían algunos incentivos o factores de motivación por los cuales se vincularía con un programa de formación en investigación? y ¿cuáles serían las áreas de investigación en las que le gustaría formular un eventual proyecto de tesis doctoral, una vez que concluya satisfactoriamente un programa de formación como este?.

Es importante mencionar que las investigaciones sobre este tema son pocas, por lo que se consideró pertinente contextualizarlo en la realidad institucional de la UNED de Costa Rica, pues resulta necesario no solo para la generación de conocimiento académico sino también para la toma de decisiones en materia de política educativa en relación con la población estudiantil, la sociedad en la que está inmersa y a la que debe responder la Universidad.

\section{Conociendo sobre el tema}

De acuerdo con Ricardo Soca, periodista y lingüista uruguayo conocido no solo por sus publicaciones editoriales sobre las palabras y sus matices, sino también por editar una página dedicada a difundir el origen de estas con el propósito de fomentar su correcto uso; señala que la palabra "doctor" es una de las más antiguas de las que existe registro. Afirma que

\begin{abstract}
Aparece por primera vez en el Libro de Alexandre, escrito a mediados del siglo XIII en el oeste de León, como dotor, con el significado de 'maestro' o 'aquel que sabe'. El primer registro escrito en español de doctor con el significado de título universitario aparece en una ley de $1462 \ldots$, aunque se la utilizó para referirse al especialista en medicina sólo a fines del siglo XVII. (1996-2014, párr.1).
\end{abstract}

La importancia de esta palabra y su significado permea a otras pues de su raíz dok, heredada de las invasiones indoeuropeas, procede, asimismo, el vocablo griego dokein, del cual vienen otras palabras tales como dogma, ortodoxia, paradoja y didáctica; y, llegadas directamente del latín, es posible encontrar docencia, doctrina, dócil y documento. Por lo tanto, no es algo que puede tomarse a la ligera ni considerarse fácil de asumir o de adquirir. 
El doctorado es un estudio formativo posible de alcanzar mediante la investigación original que permita contribuir al cúmulo de conocimiento por medio de la difusión a través de medios escritos científicos que cuenten con validez y veracidad reconocida en el ámbito al que atañe. Por ello constituye el grado de mayor relevancia por alcanzar y es sinónimo de conocimiento profundo sobre un determinado tema, cuyo objetivo primordial es la formación de investigadores.

No es de extrañar entonces, que, en un país como España, el doctorado "se entiende como el tercer ciclo de estudios universitarios oficiales, conducente a la adquisición de las competencias y habilidades relacionadas con la investigación científica de calidad”. (Real Decreto (rd) 99/2011, art. 2.1, mencionado por Jiménez, 2017, p. 127). Es así como ya no debe entenderse a partir de una simple formación universitaria, por el contrario, esta profesionalización pretende contribuir a preparar investigadores que puedan integrarse no solo en el ámbito académico docente sino también, en el mercado laboral, de manera que se crean nuevas perspectivas laborales y sociales en un mundo tan cambiante como el actual. No cabe duda de que "... los doctores deben dirigir la transferencia de conocimiento con la finalidad de contribuir a un bienestar y desarrollo de la sociedad, donde se vayan integrando en el ámbito empresarial, social y productivo considerando sus competencias investigadoras." (Jiménez, 2017, p. 129).

\section{Importancia de un doctorado}

El adquirir un título profesional dentro del escalafón establecido por la sociedad (técnico, diplomado, bachillerato, licenciatura, maestría, doctorado) constituye en sí mismo un sistema de capacitación y profesionalización de quien decide cursarlo, pero también es un indicador de mejoramiento personal en la medida en que se van adquiriendo destrezas y se van fortaleciendo competencias y habilidades. A nivel doctoral, el titularse en dicho grado proporciona capacitación en aquellos ámbitos que requieren de innovación constante, por lo que, considerando que el desarrollo investigativo es un componente fundamental para el logro de los objetivos académicos por alcanzar, un doctorando adquiere capacidades y destrezas para

desenvolverse en contextos en los que hay poca información específica; encontrar las preguntas claves que hay que responder para resolver un problema complejo; diseñar, crear, desarrollar y emprender proyectos novedosos e innovadores en su ámbito de conocimiento; trabajar tanto en equipo como de manera 
autónoma en un contexto internacional o multidisciplinar; integrar conocimientos, enfrentarse a la complejidad y formular juicios con información limitada; la crítica y defensa intelectual de soluciones. (rd 99/2011, art. 5. 2, Jiménez, 2017, p. 125).

Asimismo, la Declaración de Bergen, actividad en la cual se reunieron los ministros europeos de educación de 45 países, subraya la importancia de los doctorandos como "investigadores imprescindibles para fortalecer un conocimiento científico, original y de calidad, uniendo educación superior e investigación". (Jiménez, 2017, p. 130). Es así como un doctorado propicia la oportunidad de desempeñarse en varias trayectorias que permitan cumplir no solo con los ejes establecidos por las Universidades sino también con un mejor desarrollo social del país.

Estos esfuerzos son posibles de visualizar en las constantes publicaciones en revistas indexadas, pues en la actualidad son uno de los mayores indicadores de calidad para medir el impacto de las investigaciones elaboradas en el ámbito académico (Ruiz, López y Jiménez, 2010, p. 898). Este es uno de los componentes que permite evidenciar el trabajo constante de los investigadores, al igual que permite la obtención de reconocimiento y prestigio en el ámbito o área de trabajo en el cual se desempeña. Además, pone a disposición de la sociedad el conocimiento alcanzado y las metas logradas, así como la posibilidad de crear redes de trabajo colaborativo que puedan generar nuevos espacios investigativos en beneficio de un bien común que promueva una sociedad más evolucionada, tendiente a resolver las problemáticas o deficiencias detectadas a través de la práctica profesional, sin importar las barreras espaciotemporales.

\section{Crecimiento profesional}

El deseo de crecimiento profesional de las personas es parte de sí mismo, se aborda este tema partiendo de la premisa que cada sujeto plantea sus propias metas, logros y retos de acuerdo con sus necesidades y logra llegar a la cúspide según su nivel de motivación.

Los expertos en psicología exponen diversas teorías de la motivación, entendida como el impulso que tiene un individuo para actuar de manera que logre el objetivo propuesto; según Taype (2015) estas pueden clasificarse en dos grandes grupos: las que se enfocan en los factores internos de la persona que activan, dirigen, sostienen y detienen una conducta; y las que describen y analizan el proceso que se lleva a cabo para determinar cómo es activada, dirigida, sostenida y detenida la conducta. 
En el primer grupo se encuentra la jerarquía de necesidades de Maslow en la cual se postula que todo ser humano tiene cinco necesidades básicas: fisiológicas, de seguridad, sociales, de estima y de auto realización; seguidamente, está la teoría de McClelland quien considera que muchas de las necesidades son adquiridas en la cultura entre las que destacan la del logro, de afiliación y de poder. Por último, tenemos a Herzberg y su teoría de dos factores: insatisfactorios-satisfactorios, higiene-motivadores o extrínsecosintrínsecos; para comprender estos en el ámbito laboral los insatisfactorios, de higiene o extrínsecos son los relacionados al salario, la seguridad del puesto, las condiciones de trabajo entre otros; y los satisfactorios, motivadores o intrínsecos son el logro, el reconocimiento, el ascenso, la responsabilidad entre otros.

En el segundo grupo se encuentra Víctor Vroom con la teoría de las expectativas en la que considera que el individuo al ser alguien pensante, razonable y que alberga creencias, tiene esperanza y expectativas respecto a eventos futuros de la vida por lo que encuentra motivación en ello para realizar ciertas acciones que le permitan obtener algo específico. Le sigue la teoría de equidad de Adams, quien considera que los empleados realizan comparaciones entre los esfuerzos y recompensas propias y la de aquellos que están en condiciones similares a la suya, por lo que la percepción de equidad o injusticia se basa en los resultados obtenidos de dicha acción $\mathrm{y}$, por consiguiente, en el incremento o reducción de resultados esperados en un funcionario en específico de la organización o institución. Por último, la teoría de fijación de metas de Locke en la que declara que entre más estimulantes y retadoras sean las metas mayores niveles de desempeño por lo cual, es necesario la claridad y especificidad en la meta a razón de obtener un desempeño deseado.

A partir de lo anterior, es posible considerar que muchos profesionales se plantean la meta de obtener un grado académico que les permita cumplir con sus expectativas laborales y personales, no obstante, el tener éxito en esta línea depende tanto del individuo como del colectivo, como lo indica Chiavenato (2011) al afirmar que
el sistema de recompensas brinda diversos incentivos tanto individuales como colectivos, los cuales promueven de forma eficaz los objetivos de la organización, siendo la recompensa la mayor motivación que el o la funcionaria puede recibir por el desempeño o realización de un buen trabajo. (2011, p. 58).

Por lo tanto, considerando que el recurso humano es el mayor y mejor bien 
que una organización o institución puede tener, es importante considerar las teorías de la motivación como sustento teórico y metodológico de la estructura funcional empresarial, de manera que se pueda fomentar un crecimiento profesional eficaz y de beneficio no solo para el empleado sino también para el empleador, que potencie la sociedad en la que se desenvuelve y a la vez fortalezca el mercado nacional y mundial.

\section{Incentivos y recompensas}

Cuando se establecen nuevas metas académicas además del bien social que eso conlleva, existe un interés personal de lograr una mejor remuneración en el ámbito laboral, es decir, que se busca un reconocimiento dentro de la organización para la cual se trabaja. Los incentivos son los "pagos" de la organización a sus grupos de interés en forma de salarios, prestaciones, premios, elogios, oportunidades, reconocimiento, dividendos, entre otros (Chiavenato, 2011, p. 28); estos se otorgan a los funcionarios para motivarlos y lograr así un mejor desempeño, lo que deriva en productos de calidad para la institución. Estos varían dependiendo de la organización, el puesto y las funciones que desempeñe el personal contratado; además, están normados por reglamentos explícitos que regulan y orientan la obtención de estos.

En el caso de las recompensas se asocia a un conjunto de valores, creencias y formas de actuar del colectivo social compuesto, en el caso que ocupa, por los científicos profesionales que operan dentro y fuera de organizaciones (Fernández, Pérez y Merchán, 2005, p. 4). La naturaleza de los incentivos y las recompensas en el ámbito científico o académico no son solo económicos y se encuentran en varios niveles o grados dependiendo de los deseos que tengan los funcionarios de acuerdo con las labores asignadas al puesto, así como de los reglamentos que para este fin están definidos en la organización.

En los últimos años las recompensas se han vuelto más comunes en la línea laboral debido a que, cada vez los premios no económicos toman un auge como medio de reconocimiento por un buen desempeño, control-eficiencia de las funciones, nivel de iniciativa entre otros. Es importante considerar que este tema tiene amplia relación con el desempeño laboral pues las personas tienen ciertas expectativas que buscan alcanzar y tendrán una mejor disposición para trabajar, cumplir con las metas y los objetivos de la institución y mostrarse proactivo si todo 
ello le es retribuido con algún beneficio significativo. Es decir,

Las recompensas organizacionales, tales como pagos, promociones y otros beneficios, son poderosos incentivos para mejorar la satisfacción del empleado y su desempeño. Por ello, los expertos en Desarrollo Organizacional se preocupan cada vez más por atender el diseño e implantación de dichos sistemas. (Vargas, 2009, p. 30).

De acuerdo con Vargas (2009), los sistemas de recompensas mejoran cuatro aspectos de la eficacia organizacional: (1) motivan al personal a unirse a la organización, (2) influyen sobre los trabajadores para que acudan a su trabajo, (3) los motivan para actuar de manera eficaz y (4) refuerzan la estructura de la organización para especificar la posición de sus diferentes miembros.

Por lo tanto, existe un beneficio no solo para el funcionario y sus expectativas de crecimiento en la institución sino también para el empleador que busca operativizar de la mejor manera posible sus funciones al mismo tiempo que mantiene al mejor talento humano a su servicio. Tal y como lo explica Marcos (2011), "ejecutar una estrategia de recompensa total de forma eficaz puede incrementar la ventaja competitiva de una organización, mientras que lo contrario significa perder talento, tiempo, dinero y nuevas oportunidades". (p. 40). De esta manera, cuando una institución contempla un plan de gestión de remuneración, los funcionarios tendrán mayor ventaja competitiva debido a la motivación, el acceso a planes de mejora continua, así como un mayor cumplimiento de las metas y los logros propuestos, para beneficio bilateral de las partes.

\section{Incentivos y remuneración en la Universidad Estatal a Distancia (UNED) de Costa Rica}

De acuerdo con el Manual de Procedimientos del Proceso de Gestión de Personal de la UNED (2016), los funcionarios de dicha institución cuentan con los siguientes beneficios directos: las anualidades, el reconocimiento de experiencia en el sector público, el adelanto de salario, el descuento y crédito, la modificación de horario laboral, ayudas especiales (gastos de funeral) y las vacaciones (institucionales y flotantes). En cuanto a los indirectos se identificó la salud laboral y la asociación solidarista. (p. 12).

Cada uno de ellos está normado por una serie de aspectos que se deben cumplir, pero, además, especificados de manera tal que el funcionario conozca cuál será el claro beneficio que adquirirá. Del mismo modo, este manual especifica que los incentivos directos que podrá recibir son la participación en cursos y seminarios 
y el reconocimiento de unidades paso en puestos no profesionales (p. 15).

Otro incentivo que se puede alcanzar en la Universidad es mediante la promoción profesional en la cual, se otorgan puntos para ascender en Carrera Profesional y así obtener un aumento significativo de un porcentaje específico sobre la base salarial. Para ello, se consideran aspectos tales como: condición académica, experiencia profesional o académica en la UNED, experiencia profesional o académica fuera de la UNED, publicaciones, conocimiento de idiomas extranjeros o lenguaje de computación, premios y honores y; obras artísticas o técnicas (p. 6-7), según lo normado en el artículo 13 del Reglamento de Carrera Universitaria.

Además, el artículo 15 de este mismo reglamento estipula la siguiente valoración con relación al aspecto de condición señalado con anterioridad:

Tabla 1: Promoción profesional mediante puntos.

\begin{tabular}{c|c}
\hline Grado académico & Puntos \\
\hline Diplomado Universitario & 4 \\
\hline Bachillerato Universitario & 6 \\
\hline Licenciatura & 10 \\
\hline Especialidad profesional de postgrado & 12 \\
\hline Maestría o grado académico equivalente & 14 \\
\hline Doctorado académico & 18 \\
\hline
\end{tabular}

Fuente: Elaboración propia

Con base en lo anterior, para que un funcionario ascienda profesionalmente, debe cumplir con los siguientes requisitos:

Tabla 2: Requisitos para ascender profesionalmente en la UNED.

\begin{tabular}{|c|c|c|c|c|c|}
\hline Requisito & Profesional 1 & Profesional 2 & Profesional 3 & Profesional 4 & Profesional 5 \\
\hline $\begin{array}{l}\text { Condición } \\
\text { académica y laboral }\end{array}$ & $\begin{array}{l}\text { Bachiller } \\
\text { Universitario y } \\
\text { experiencia la } \\
\text { requerida de } \\
\text { acuerdo con el } \\
\text { Manual de } \\
\text { Puestos. }\end{array}$ & $\begin{array}{l}\text { Licenciatura y } \\
\text { experiencia la } \\
\text { requerida de } \\
\text { acuerdo con el } \\
\text { Manual de } \\
\text { Puestos. }\end{array}$ & $\begin{array}{l}\text { Licenciatura y } \\
3 \text { años de } \\
\text { experiencia ó } \\
\text { Maestría y } 1 \\
\text { año de } \\
\text { experiencia }\end{array}$ & $\begin{array}{c}\text { Licenciatura y } \\
5 \text { años de } \\
\text { experiencia } \\
\text { laboral } 3 \text { de } \\
\text { ellos en la } \\
\text { UNED ó } \\
\text { Maestría y } 3 \\
\text { años de } \\
\text { experiencia } 2 \\
\text { de ellos en la } \\
\text { UNED o }\end{array}$ & $\begin{array}{l}\text { Licenciatura y } \\
15 \text { años de } \\
\text { experiencia } \\
\text { laboral } 5 \text { de } \\
\text { ellos en la } \\
\text { UNED ó } \\
\text { Maestría y } 12 \\
\text { años de } \\
\text { experiencia } 4 \\
\text { de ellos en la } \\
\text { UNED o }\end{array}$ \\
\hline
\end{tabular}




\begin{tabular}{|c|c|c|c|}
\hline & & $\begin{array}{c}\text { Doctorado y 2 } \\
\text { años de } \\
\text { experiencia 1 } \\
\text { de ellos en la } \\
\text { UNED }\end{array}$ & $\begin{array}{c}\text { Doctorado y 10 } \\
\text { años de } \\
\text { experiencia 3 } \\
\text { de ellos en la } \\
\text { UNED }\end{array}$ \\
\hline $\begin{array}{c}\text { Publicaciones, } \\
\text { premios y honores } \\
\text { por obras artísticas, } \\
\text { científicas o } \\
\text { técnicas, } \\
\text { reconocimientos }\end{array}$ & Mínimo 6 & Mínimo 12 \\
puntos & & \\
\hline $\begin{array}{c}\text { Idioma extranjero, } \\
\text { lenguaje } \\
\text { computacional, } \\
\text { LESCO o bien } \\
\text { lenguas de pueblos } \\
\text { originarios* }\end{array}$ & & & \\
\hline
\end{tabular}

Fuente: Elaboración propia

* Cuando el idioma o lenguaje sea requisito del puesto, no se acreditarán puntos.

Nótese en la tabla anterior, que el grado académico de doctorado no es requisito indispensable para ascender en carrera profesional, lo cual, si bien brinda las mismas posibilidades para los demás grados académicos, considerando la importancia que otorga el doctorando para la institución, este no cuenta con mayor diferenciación respecto de su incentivo salarial en comparación con una licenciatura, por ejemplo.

Por otro lado, en relación con los incentivos que brinda la Universidad, los funcionarios que desean cursar un postgrado pueden optar por una beca. La institución puede otorgar financiamiento parcial o total, siempre y cuando cumpla con los objetivos del Plan Operativo Anual (POA) de la dependencia o programa en la que labora el o la funcionaria y las disponibilidades

presupuestarias correspondientes, de acuerdo con el Reglamento de becas para la Formación y la Capacitación del Personal de la Universidad Estatal a Distancia (UNED). Además, en el artículo 10, se especifican las ayudas económicas no reembolsables que se pueden asignar a los funcionarios para cubrir los siguientes rubros: (1) derecho de matrícula, (2) pago de materias, según el período establecido para ello, (3) costo de los libros de texto, siempre que no exceda el valor conjunto de la matrícula, (4) tesis o trabajos finales de graduación (TFG), exámenes o seminarios de grado, exámenes de candidatura, entre otros, (5) hospedaje, (6) pasajes y viáticos de ley, de acuerdo con la normativa correspondiente, (7) manutención de familiares hasta $50 \%$ para el cónyuge o pareja de hecho, según el 
Código de Familia, constituida antes de otorgar la beca y hasta $25 \%$ para cada hijo(a) hasta un máximo de dos. Estos porcentajes se calculan sobre la base de la mensualidad aprobada para el becario y de conformidad con los criterios, los requisitos y los procedimientos que el Consejo de Becas Institucional (COBI) defina y (8) cualquier otro no previsto en los puntos anteriores y acordados por el COBI relacionado directamente con la formación o la capacitación del funcionario correspondiente.

Además de lo señalado, se otorga el beneficio de licencia o permiso con goce de salario para participar de planes de formación, capacitación y pasantías (artículo 7, inciso b).

\section{Metodología}

Considerando la importancia que tienen los estudios doctorales para la producción investigativa, así como el crecimiento y la formación del profesional que se decide cursar, el Programa de Investigación en Fundamentos de la Educación a Distancia (PROIFED) realizó un estudio fundamentado en el paradigma naturalista y holístico; con el objetivo de analizar el interés para la formación de alto nivel en investigación, conducente a un doctorado académico según los diferentes campos disciplinares, de manera que fuera posible realizar un mapeo del posicionamiento de los funcionarios de la institución interesados en la obtención de un título académico en el grado de doctor.

De acuerdo con Hernández, Fernández y Baptista (2014) el enfoque de este tipo de estudio es cuantitativo, de profundidad descriptiva y basado en trabajo de campo. Con esto se busca especificar las propiedades, características y perfiles de personas, grupos, comunidades, procesos, objetos o cualquier fenómeno que se someta a un análisis; en el caso particular de este estudio, a 892 funcionarios de la Universidad Estatal a Distancia (UNED) de Costa Rica, los cuales tienen grado de Máster. Dicha base de datos fue suministrada por la Oficina de Recursos Humanos de la institución.

La obtención de la información para esta investigación se planteó en dos etapas: el análisis de documentación y la aplicación de un instrumento a una población determinada. En la primera etapa se realizó una búsqueda en diferentes bases de datos (ESBCO, Google Académico, entre otros), además de consultar la normativa, manuales y documentación institucional de la Universidad, partiendo de una serie de palabras vinculantes a la temática por desarrollar. 
De acuerdo con Barrantes (2014), “el análisis de contenido es un proceso que por medio de características relevantes del contenido de un mensaje permite la transformación a unidades que dan pie a la descripción y análisis de las variables planteadas". (p. 283); para ello se ordenó de forma alfabética los documentos seleccionados de acuerdo con el título y se procedió a elaborar una matriz conformada por una serie de criterios entre los que están el número de fuente, autor, tema de interés, página y cita textual; para así lograr obtener el sustento teórico de las variables de incentivos, recompensas, doctorado, desarrollo profesional, investigación, motivación entre otras.

A continuación, se muestra un extracto de la matriz elaborada:

Tabla 3: Extracto de la matriz elaborada para el análisis de contenido.

\begin{tabular}{|c|c|c|c|c|}
\hline $\begin{array}{c}\text { Número } \\
\text { fuente }\end{array}$ & Autores & $\begin{array}{c}\text { Tema de } \\
\text { interés }\end{array}$ & Pág. & Cita textual \\
\hline 1 & Chiavenato, I & Incentivos & 28 & $\begin{array}{c}\text { Los incentivos son los "pagos" de la organización } \\
\text { a sus grupos de interés en forma de salarios, } \\
\text { prestaciones, premios, elogios, oportunidades, } \\
\text { reconocimiento, dividendos, entre otros }\end{array}$ \\
\hline
\end{tabular}

Fuente: Elaboración propia.

En la segunda etapa se aplicó un cuestionario en línea con 17 preguntas, de las cuales 15 fueron cerradas y 2 abiertas; dicho instrumento fue validado por el equipo profesional del PROIFED, entre los que se encontraron filólogos, psicólogos, docentes, literatos, lingüista, administradores y sociólogos; estos investigadores tienen entre 5 y 15 años de experiencia en el desarrollo de iniciativas de investigación. El instrumento se programó con la herramienta LimeSurvey y se envió a los funcionarios mediante un enlace web al correo electrónico registrado, y estuvo disponible del 09 al 31 de mayo de 2019, con recordatorios enviados el 20 y 28 de mayo de 2019.

Se procesó la información brindada mediante una matriz de cálculo, en donde aplicando la estadística descriptiva para cada variable planteada se le determinó la frecuencia, el porcentaje acumulado, así como las medidas de tendencia central y de variabilidad, representando los resultados en tablas con los valores obtenidos.

\section{Análisis de resultados}

La base de datos facilitada fue de 892 personas, de las cuales 80 no recibieron el correo electrónico por diversas situaciones entre ellas: se 
encontraban pensionadas, actualmente no trabajaban en la universidad, o la cuenta de correo no se encontró en el servidor; de 508 no se obtuvo respuesta. Se recibieron un total de 304 cuestionarios completos en los cuales los participantes indicaron los incentivos para cursar un doctorado. Con base en ello, a continuación, se detallan algunos de los resultados obtenidos.

Sobre la información básica de la población de estudio, el $67,11 \%$ trabajan en el área de docencia, el $17,11 \%$ en el área administrativa, un $9,54 \%$ en investigación, un 4,61\% en extensión y el restante $1.64 \%$ de la población no brindó respuesta. En relación con el tipo de nombramiento se encontró que un 56,91\% cuenta con propiedad, un $37,33 \%$ se encuentra nombrado a plazo fijo y un $3,62 \%$ en servicios especiales y el $1.64 \%$ de la población no brindó respuesta.
Respecto a la jornada de trabajo un 58,88\% trabaja 42,5 horas a la semana lo que equivale a tiempo completo y el $41,12 \%$ restante labora un promedio de entre $21 \mathrm{y}$ 10,5 horas semanales, lo que corresponde a medio tiempo y un cuarto de tiempo respectivamente.

La población encuestada se encuentra compuesta por 170 mujeres lo que es equivalente al 55,92\% del total, 130 personas son hombres para un $42,76 \%$ y un $1,32 \%$ no indicaron su sexo. El rango de edades ronda entre los 40 o más años para un $75,33 \%$, el $22,70 \%$ tienen entre 30 y 39 años, un $1,64 \%$ no dieron respuesta y el porcentaje más bajo con un $0,33 \%$ es el rango de 20 a 29 años.

Tabla 4: Modalidad de interés del programa.

\begin{tabular}{|c|c|c|}
\hline Opciones de respuesta & Cantidad de respuestas & Porcentajes \\
\hline Totalmente a distancia & 53 & $17,43 \%$ \\
\hline Híbrido (virtual y presencial) & 153 & $50,33 \%$ \\
\hline Totalmente virtual & 95 & $31,25 \%$ \\
\hline Sin respuesta & 3 & $0,99 \%$ \\
\hline Total & 304 & $100,00 \%$ \\
\hline
\end{tabular}

Fuente: Elaboración propia.

En cuanto a la modalidad de interés del programa que les gustaría llevar, la tabla 4 muestra que un 50,33\% de los participantes tiene mayor interés por una oferta híbrida, seguido de un $31,25 \%$ que prefiere que sea totalmente virtual; un $17,43 \%$ se inclina por un estilo totalmente a distancia $y$, finalmente, un $0,99 \%$ no dio respuesta. Nótese que, a pesar de estar la modalidad totalmente virtual como una 
opción de respuesta, la selección de esta respuesta no alcanza el porcentaje mayor, es decir, que quizá exista una relación entre el rango de edad y la preferencia de la modalidad pues son los de 40 años o más quienes tuvieron interés en participar del estudio. Es decir, la población más interesada en cursar un programa doctoral es la que se encuentra en una etapa más madura de su adultez y que, además, no sean nativos digitales, por lo que prefieren una convergencia entre lo presencial y lo virtual.

Tabla 5: Condiciones laborales necesarias para la vinculación.

\begin{tabular}{c|c|c|}
\hline \multicolumn{1}{c}{ Opciones de respuesta } & Cantidad de respuestas & Porcentaje \\
\hline $\begin{array}{c}\text { Tiempo de estudio } \\
\text { (¿Cuántas horas a la semana?) }\end{array}$ & 179 & $58,88 \%$ \\
\hline Apoyo de la jefatura inmediata & 83 & $27,30 \%$ \\
\hline Otro & 39 & $12,83 \%$ \\
\hline Sin respuesta & 3 & $0,99 \%$ \\
\hline Total & 304 & $100,00 \%$ \\
\hline
\end{tabular}

Fuente: Elaboración propia.

A la población encuestada se le consultó cuáles consideran son las condiciones laborales necesarias para vincularse en un programa de doctorado y tal como se muestra en la tabla 5 , en su mayoría mencionan que el tiempo de estudio es fundamental o la asignación de horas estudio dentro de su carga laboral con un $58,88 \%$, seguido de un $27,30 \%$ que necesita el apoyo de la jefatura inmediata; el $12,83 \%$ brinda diferentes aspectos necesarios para poder cursar el programa como, por ejemplo: teletrabajo, estabilidad laboral-salarial (nombramiento), permisos para poder desarrollar las actividades académicas, permiso con goce de salario, flexibilidad en los horarios, recurso económico-físico-de inversión, apoyo en el caso de enfermedad o situaciones de salud o familiares, apoyo con un asistente, maya curricular flexible y por último, incentivos laborales. Un 0,99\% no da respuesta especifica de la preferencia.

La población que indicó que el tiempo es fundamental, dieron los rangos de horas que consideran podrían invertir por semana al estudio, obteniendo un $41,12 \%$ que plantea entre 19 y 24 horas, el $25,46 \%$ se inclina entre 1 a 6 horas, el 25, $32 \%$ necesitaría entre 7 a 12 horas, el $5,02 \%$ indica ocupar un rango entre 25 a 48 horas y solo un $3,09 \%$ señala que entre 13 a 18; tal y como se muestra en la tabla 6. Es importante considerar que, para cada asignatura de la maya curricular, existe un rango de horas de estudio específico para 
dedicarle, misma que podría no estar encuestados, lo que puede originar coincidiendo con lo planteado por los eventuales abandonos de la oferta.

Tabla 6: Cantidad de horas a dedicar al doctorado.

\begin{tabular}{c|c|c|}
\hline \multicolumn{1}{|c|}{ Cantidad de horas } & Cantidad de respuestas & Porcentajes \\
\hline 1 a 6 & 33 & $25,46 \%$ \\
\hline 7 a 12 & 30 & $25,32 \%$ \\
\hline 13 a 18 & 6 & $3,09 \%$ \\
\hline 19 a 24 & 47 & $41,12 \%$ \\
\hline 25 a 48 & 7 & $5,02 \%$ \\
\hline Total & 123 & $100,00 \%$ \\
\hline
\end{tabular}

La tabla 7 muestra información sobre los incentivos o factores de motivación que impulsaría a los participantes a vincularse a un doctorado, entre los que se encuentran: el desarrollo de proyectos que causen impacto social con un $13,18 \%$, ser parte del cuerpo docente o de los asesores de investigación del doctorado al culminar el programa con un $10,71 \%$. Un $10,42 \%$ quisiera unirse a un programa o laboratorio de investigación nacional o internacional relacionado con su área de interés, o bien vincularse a redes relacionadas con el doctorado el $7,89 \%$ de los encuestados. Además, un $12,95 \%$ indican que la participación en pasantías fuera del país en centros de investigación es un incentivo importante.

En cuanto a la línea de incentivos económicos el $12,89 \%$ de los participantes se inclinan por el hecho de contar con un permiso con goce de salario (tener tiempo para cursar la iniciativa siempre que este tiempo sea pagado). El 8,53\% indica que el aspecto salarial es parte importante por lo que esperaría que los ingresos percibidos se reflejen con una mejora o aumento de salario. Entre los incentivos personales la población participante se inclina en un $8,71 \%$ al ascenso o crecimiento profesional, además el $7,71 \%$ de los encuestados aspira a liderar áreas de investigación en las dependencias en las que laboran. Por otra parte, un 5,59\% contempla el reconocimiento institucional (contar con renombre tanto en la Universidad como en otras instituciones educativas) y solo el $1,41 \%$ tienen otra serie de incentivos en mente. 
Tabla 7: Incentivos o factores de motivación.

\begin{tabular}{|c|c|c|}
\hline Opciones de respuesta & $\begin{array}{l}\text { Cantidad de } \\
\text { respuestas }\end{array}$ & Porcentajes \\
\hline Permiso con goce de salario para estudiar e investigar & 219 & $12,89 \%$ \\
\hline $\begin{array}{l}\text { Vinculación en un programa o laboratorio de investigación nacional } \\
\text { e internacional relacionado con su área de interés }\end{array}$ & 177 & $10,42 \%$ \\
\hline Participar en pasantías fuera del país en centros de investigación & 220 & $12,95 \%$ \\
\hline $\begin{array}{l}\text { Ser parte de los profesores o asesores de investigación del } \\
\text { doctorado al culminar el programa }\end{array}$ & 182 & $10,71 \%$ \\
\hline Vinculación en diferentes redes asociadas al doctorado & 134 & $7,89 \%$ \\
\hline Liderar áreas de investigación & 131 & $7,71 \%$ \\
\hline Desarrollo de proyectos que causen impacto social & 224 & $13,18 \%$ \\
\hline Mejora de situación salarial & 145 & $8,53 \%$ \\
\hline Ascenso profesional & 148 & $8,71 \%$ \\
\hline Reconocimiento institucional & 95 & $5,59 \%$ \\
\hline Otro & 24 & $1,41 \%$ \\
\hline Total & 1699 & $100,00 \%$ \\
\hline
\end{tabular}

Fuente: Elaboración propia.

Como es posible notar, la mejora en el aspecto salarial o el ascenso profesional no figuran como las opciones con un alto porcentaje de respuesta en esta consulta en particular, por el contrario, figuran con mayor valor las correspondientes al desarrollo de proyectos con impacto social y la participación en pasantías a centros de investigación; es decir, que para aquellos que desean cursar un programa doctoral tiene más significancia el impacto social y académico que se pueda generar que la mejora salarial.

Lo anterior, podría estar vinculado con el rango de adultez de quienes contestaron el instrumento, así como de la permanencia en la Universidad y su grado de pertenencia a ella. $\mathrm{Si}$ bien la profesionalización brinda la posibilidad de un incremento salarial, esto no es posible de garantizar en todas las instituciones y en el caso particular de la UNED no es siquiera requisito indispensable para el ascenso en carrera profesional por lo que, los mismos funcionarios deben estar familiarizados con esto y no lo tienen como uno de los factores más importantes a considerar.

Respecto al $1,41 \%$ del rubro denominado Otro se mencionaron aspectos como: crecimiento personal y profesional, obtener más herramientas para ser mejor profesional y docente, aprendizaje, fortalecer la Extensión Universitaria, aportar a la Universidad conocimiento, incrementar los índices de impacto académico de la UNED y; actualización profesional y académica que mejore los procesos docentes e investigativos. 


\section{Conclusiones}

El 55,92\% de la población participante interesada son mujeres, de las cuales un $37,33 \%$ de los 304 encuestados cuenta con propiedad. Esta población se compone de 229 personas con un rango de edad que oscila entre los 40 años o más, lo que representa una trayectoria laboral y académica muy amplia, debido a que todos cuenta con un grado académico de Máster.

Los participantes interesados en poder llevar el doctorado prefieren una modalidad educativa híbrida (presencial y virtual) con el uso de plataformas académicas y, a menos del $31 \%$ les llamó la atención que el programa se desarrolle por completo de manera virtual.

Un aspecto fundamental para participar en un doctorado son las condiciones laborales que le puede brindar la institución al interesado, en este caso la Universidad, en donde el 58,88\% de la población indican que las horas de estudios son de suma importancia para dicha vinculación; entre los rangos de horas que se mencionaron para invertir se encuentra que más del $40 \%$ necesitaría un promedio de 19 a 24 horas semanales, las cuales deberían ser consensuadas entre la jefatura inmediata y el funcionario, a la luz de la reglamentación que exista sobre este aspecto.
El $27,30 \%$ de la población ve necesario el apoyo incondicional de la jefatura inmediata con el fin de poder auto gestionar tiempos para la entrega de productos y la autorización para participar en las actividades académicas que demanda el programa de doctorado, así como la agilización de los trámites requeridos para tal fin.

Otros aspectos importantes para la vinculación serían la posibilidad del teletrabajo como medio para evitar perder tiempo en el desplazamiento casa-trabajo y viceversa, una mejor estabilidad laboral, salarial (nombramiento), contar con los permisos para poder desarrollar las actividades académicas, permiso con goce de salario, flexibilidad en los horarios y algunos otros incentivos laborales que beneficiarían la consecución del compromiso y la responsabilidad que conlleva el cursar un doctorado.

El estudio logró determinar que las recompensas o factores de motivación con mayor apreciación por parte de los participantes se relacionan, en su mayoría, con el planteamiento de proyectos con beneficio social, así como la vinculación a diferentes redes de investigación tanto nacionales como internacionales, dejando relegada la opción relacionada con el posible aumento salarial. Es decir, que los participantes del estudio consideran que se 
obtiene mayor estímulo y distinción en las labores asociadas directamente con la investigación e innovación que se desprenden del doctorado, que un porcentaje de aumento salarial, lo que se refleja en los comentarios relacionados al crecimiento personal y profesional, obtener más herramientas para ser mejor profesional y docente, fortalecer la extensión universitaria y aportar a la Universidad conocimiento; lo que genera un sentido de pertenencia a la institución sumamente valioso.

\section{Recomendaciones}

Es importante que la Universidad se plantee la posibilidad de ascenso en carrera profesional con la obtención del título de doctorado, considerando que la inversión de trabajo que realizó el graduado es ardua y la sola titulación representa un gran logro no solo para quien lo ostenta sino también para la institución misma, pues abre la oportunidad a más y mejores líneas de investigación e innovación; lo que se vería retribuido no solo en la vinculación académica que detallan los participantes sino también en el aporte económico que el doctorando podría recibir para así invertir en su beneficio profesional y personal, otorgándole mayor motivación para mantenerse en la institución.
Asimismo, implementar una estrategia que permita la generación de profesionales más capacitados en el área de investigación, de manera que se puedan generar proyectos investigativos innovadores y pertinentes para la sociedad actual; mismos que podrían ser coordinados por los doctores académicos, ya que se consideran profesionales idóneos para la gestión, investigación y toma de decisiones. Un modelo estratégico que se podría implementar es el Spin-off, considerado pertinente para la captación de nuevos recursos, vinculación con los sectores público y privado, generador de iniciativas y obtención de una serie de recompensas.

Por otro lado, incentivar más a la población académica a que participe de los programas de doctorado pues los beneficios que se pueden llegar a obtener serían bilaterales, pues por un lado está el renombre, en un área específica, que el doctorando puede llegar a adquirir, y por el otro, este reconocimiento tendría un reflejo en la Universidad al ser un funcionario de esta. Por lo tanto, podrían tenerse al alcance patrocinios tanto para el investigador como para la institución, pues no se debe olvidar que el propósito de cursar un doctorado es la de transferir conocimiento en beneficio y desarrollo del bien común, de manera que haya una 
sinergia entre el ámbito empresarial, el social y el productivo.

Es fundamental que la Universidad incentive a sus funcionarios para que se profesionalicen aún más a través de la incursión de doctorados, de manera que puedan concretarse más y mejores proyectos de investigación que fortalezcan el quehacer de la institución, así como beneficiar a la sociedad en la que se encuentra inmersa ya que, es a través del área investigativa que se buscan soluciones factibles a los problemas que surgen día con día.

\section{Referencias}

Barrantes, E. (2014). Investigación: Un camino al conocimiento, un enfoque cualitativo, cuantitativo y mixto. $2^{\mathrm{a}}$ Edición. Costa Rica: EUNED.

Chiavenato, I. (2011). Comportamiento organizacional. La dinámica del éxito en las organizaciones. $2^{\text {a }}$ Edición. México: Mc Graw Hill Educación.

Fernández, M., Pérez, M., \& Merchán, C. (2005). El sistema de incentivos y recompensas en la ciencia pública española. [Documento de trabajo]. Recuperado de http://digital.csic.es/handle/10261/2044

Hernández, R., Fernández, C., \& Baptista, P. (2014). Metodología de la Investigación. México: Mc Graw Hill.

Jiménez, M. (2017). Los nuevos estudios de doctorado en España: avances y retos para su convergencia con Europa. Revista Iberoamericana de Educación Superior,
8(21), 123-137. Recuperado de http://www.scielo.org.mx/scielo.php?pid= S2007-

$28722017000100123 \&$ script $=$ sci_abstract \&tlng=pt

Marcos, S. (Octubre, 2011). Recompensa total: el nuevo contrato. Harvard Deusto business review, (206), 40-51. Recuperado de https://www.harvarddeusto.com/recompensa-total-el-nuevo$\underline{\text { contrato }}$

McClelland, D. (1961). The achieving society. Princeton: Van Nostrand Reinhold.

Ruiz, R., López, E. D., \& Jiménez, E. (2010). Principios y criterios utilizados en España por la Comisión Nacional Evaluadora de la Actividad Investigadora (CNEAI) para la valoración de las publicaciones científicas: 19892009. Psicothema, 22(4), 898-908.

Soca, R. (1996-2014). Doctor. La página del idioma español. Recuperado de http://www.elcastellano.org/palabra/doctor

Taype, M. (2015). Teorías de la motivación. Recuperado de https://www.gestiopolis.com/teorias-de-lamotivacion/

Universidad Estatal a Distancia. (2010). Reglamento de becas para la formación y la capacitación del personal de la UNED. Recuperado de https://www.uned.ac.cr/images/ami/docum entos/Reglamento\%20COBI.PDF

Universidad Estatal a Distancia. (2016). Manual de Procedimientos del Proceso de Gestión de Personal de la UNED. Recuperado de https://www.uned.ac.cr/viplan/images/cppi /UNED-MPGA-PGP.01_-

Proceso_de_Gesti\%C3\%B3n_del_Personal .pdf 
Universidad Estatal a Distancia. (2018). Reglamento de Carrera Universitaria. Recuperado de https://www.uned.ac.cr/academica/images/ cidreb/reglamento/admin-recursos humanos/Carrera_Universitaria_18_enero$\underline{\text { 2018.pdf }}$

Vargas, A. (2009). Importancia del factor humano en el diseño de sistemas de recompensas: Capítulo 5. Sistemas de recompensas (Tesis de Grado). Universidad de Sonora, México. Recuperado de http://www.bidi.uson.mx/tesisDet.aspx?crit $1=$ TITULO\&texto1=sistemas\%20de\%20re compensa\&crit $2=$ TITULO\&texto $2=$

\author{
Información del articulo / Article Information \\ Recibido en: 26/02/2020 \\ Aprobado en: 07/04/2020 \\ Publicado en: 29/05/2020 \\ Received on February 26th, 2020 \\ Accepted on April 07th, 2020 \\ Published on May, 29th, 2020 \\ Contribuciones en el artículo: Las autoras fueron \\ responsables de todas las etapas y resultados de la \\ investigación, a saber: elaboración, análisis e \\ interpretación de datos; escribir y revisar el contenido del \\ manuscrito y; aprobación de la versión final publicada.
}

Author Contributions: The author were responsible for the designing, delineating, analyzing and interpreting the data, production of the manuscript, critical revision of the content and approval of the final version published.

Conflictos de Intereses: Las autoras han declarado que no existe conflicto de intereses con respecto a este artículo.

Conflict of Interest: None reported.

Orcid

Karla Yanitzia Artavia Díaz

http://orcid.org/0000-0003-1337-3466

Alejandra Castro Granados

http://orcid.org/0000-0003-2110-934X

\section{Cómo citar este artículo / How to cite this article}

APA

Artavia Díaz, K. Y., \& Castro Granados, A. (2020). Los estudios doctorales: crecimiento profesional por sobre los incentivos organizacionales. Un estudio en la Universidad Estatal a Distancia (UNED) de Costa Rica. Rev. Bras. Educ. Camp., 5, e8519. http://dx.doi.org/10.20873/uft.rbec.e8519

ABNT

ARTAVIA DÍAZ, K. Y.; CASTRO GRANADOS, A. LoS estudios doctorales: crecimiento profesional por sobre los incentivos organizacionales. Un estudio en la Universidad Estatal a Distancia (UNED) de Costa Rica. Rev. Bras. Educ. Camp., Tocantinópolis, v. $5, \quad$ e8519, 2020. http://dx.doi.org/10.20873/uft.rbec.e8519 